\title{
ReMed: Jahresbericht 2020
}

\section{ReMed-Leitungsausschuss}

\author{
Ärztinnen und Ärzte kümmern sich um andere und fokussieren auf die Bedürf- \\ nisse ihrer Patientinnen und Patienten. Es kommt jedoch vor, dass sie selbst auch \\ von Schwierigkeiten und gesundheitlichen Problemen betroffen sind. In der \\ Schweiz gibt es seit 2007 das Unterstützungsnetzwerk ReMed für in der Schweiz \\ tätige Ärztinnen und Ärzte, die sich in einer Krise befinden.
}

\section{Das Unterstützungsnetzwerk ReMed ist für Sie da}

Das ReMed bietet verschiedene Angebote, die speziell auf die Bedürfnisse der Ärzteschaft ausgerichtet sind. Innerhalb von 72 Stunden steht Ihnen ReMed unterstützend zur Seite. Der Kontakt zu ReMed kann auch über Personen aus dem Umfeld der betroffenen Ärztinnen und Ärzte erfolgen. Die Erhaltung der Gesundheit und ärztlichen Funktionalität sowie die Gewährleistung der Patientensicherheit und der hohen Qualität in der medizinischen Versorgung stehen dabei an erster Stelle.

\section{Kontaktaufnahmen im Jahr 2020}

Im vergangenen Jahr wurde ReMed 170-mal kontaktiert. Die Anfragen sind somit gegenüber dem Vorjahr um 5\% gestiegen. Die stetige Zunahme der Kontaktaufnahmen zeigt die Dringlichkeit der ReMed-Dienstleis-

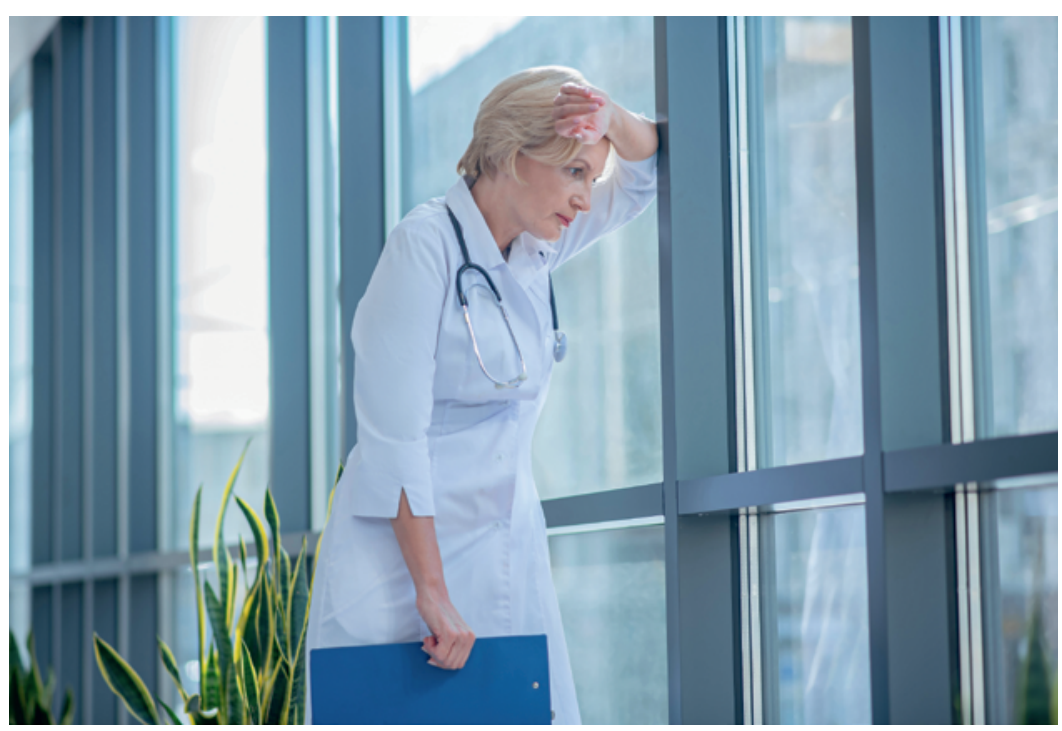

ReMed unterstützt Ärztinnen und Ärzte, die an die Grenze ihrer physischen und mentalen Kräfte gelangt sind. tungen deutlich. Die Anfragen kamen zu 64\% aus der Deutschschweiz, zu 34\% aus der Romandie und zu 2\% aus der italienischen Schweiz. Wie bereits im Vorjahr waren die Kontaktsuchenden zu 60\% weiblich und zu 40\% männlich. Die strukturellen Probleme (u.a. Belastung am Arbeitsplatz, administrative Überbelastung), psychische Beeinträchtigungen sowie Angst (u.a. Zukunftsängste, Laufbahnentscheide, Situation vor Praxisab- und -übergabe, Angst vor Behandlungsfehlern) waren die Hauptgründe der Kontaktaufnahme zum Unterstützungsnetzwerk.

\section{Erweitertes Angebot während der Corona-Krise}

ReMed hat in der Coronavirus-Krise das Angebot erweitert und die personellen Ressourcen verstärkt. Damit unterstützte das Programm Ärztinnen und Ärzte, welche in dieser besonderen Situation an die Grenze ihrer physischen und mentalen Kräfte gelangt sind. So wurden aufgrund der aktuellen Corona-Pandemie im Jahr 2020 in der Deutschschweiz erstmals moderierte Online-Coaching-Gruppen zum lösungsfokussierten, interkollegialen Austausch über die aktuellen Herausforderungen angeboten. Ein kleines Team von Ärzten entwickelte während der drei moderierten Video-Meetings mit Hilfe des lösungsfokussierten Kurzzeit-Coachingansatzes ein einfaches Tool, um die aktuellen Belastungsfaktoren der Assistenzärztinnen und -ärzte in ihrer Abteilung im Spitalalltag erfolgreich abzuholen und damit Überlastungen proaktiv vorzubeugen.

\section{Spontan-Coachinggruppen für Spitalärztinnen und -ärzte}

ReMed führte 2020 erstmals im Rahmen eines Pilotprojektes Spontan-Coachinggruppen für Spitalärztinnen und Spitalärzte in Zürich durch. Unter stren- 


\section{Angebote der FMH zur Ärztegesundheit}

\section{Angebot \\ ReMed}

Coach my Career

Prevention for doctors

Berufsentwicklung
Kurzbeschrieb

Unterstützungsnetzwerk für Krisensituationen sowie die Vermittlung von präventivem Wissen und Erfahrung mit 24-StundenHelpline: www.swiss-remed.ch

Generationsübergreifendes Mentoring-Angebot für den ärztlichen Nachwuchs, insbesondere für die Planung der Weiterbildung und beruflichen Entwicklung

Veranstaltungen zur Sensibilisierung für einen adäquaten Umgang mit Belastungen des Ärztealltages. Leitung der breit abgestützten Arbeitsgruppe zur Umsetzung von diversen Projekten

Analyse der Bedürfnisse der ärztlichen Nachwuchskräfte und Beobachtung des medizinisch-demographischen Wandels. Entwicklung neuer Dienstleistungen für die Mitglieder der FMH

gen Covid-19-Hygiene- und -Sicherheitsmassnahmen konnten vier Coachinggruppen mit jeweils zwei bis drei Assistenzärzt(inn)en in den Räumlichkeiten des Universitätsspitals Zürich durchgeführt werden. Die Leitung der Gruppen hatten Vertreter des Leistungsausschusses inne. Die Spontan-Coachinggruppen sollen 2021 weitergeführt werden.

\section{Evaluation der ReMed-Unterstützungs- tätigkeit mit Fokus auf Beratungsqualität}

Die Qualitätssicherung und die Entwicklung des Angebots ReMed waren und sind dem Leitungsausschuss ein zentrales Anliegen. In einem ersten Schritt wurde deshalb bereits 2015 eine Evaluation des Programmes extern in Auftrag gegeben (Ettlin, Grunder, Jörg \& Widmer, 2016). Es handelte sich dabei um eine Evaluation der Strukturqualität. Der Fokus der damaligen Evaluation lag auf dem Budget, der Infrastruktur und dem Personal. Die Untersuchung erfolgte mittels Inter-

\section{ReMed hat in der Coronavirus-Krise das Ange- bot erweitert und die personellen Ressourcen verstärkt.}

views, Online-Befragung und der Analyse der bestehenden Grundlagendokumente von ReMed. Im Bericht waren Handlungsempfehlungen formuliert, welche $a b$ 2016 sukzessive umgesetzt wurden. So wurden beispielsweise neue Personen für den Leitungsausschuss rekrutiert (Integration aller Sprachregionen), die Abläufe der Fallverteilungen effizienter gestaltet, das Handbuch überarbeitet und damit auch die Begrifflichkeiten der Angebote angepasst sowie Massnahmen getroffen, um die Bekanntheit von ReMed zu steigern.
Das primäre Ziel der aktuellen Evaluation ist die Analyse der Beratungsqualität. Vom 1. November 2018 bis 30. April 2020 nahmen 120 Ärztinnen und Ärzte (Rücklauf 42\%), welche das Angebot von ReMed in Anspruch nahmen, an der Evaluation teil. Die persönlichen Daten und die Krankengeschichte waren zu keinem Zeitpunkt Teil der vorliegenden Evaluation.

Es zeigte sich, dass die Strukturqualität zu 97\%, die Prozessqualität zu 90\% und die Ergebnisqualität zu $82 \%$ positiv bewertet wurden.

Die sehr gute Beurteilung der Qualität des Beratungsangebots durch die Ratsuchenden lässt den Schluss $\mathrm{zu}$, dass die regelmässige externe Überprüfung der eigenen Arbeit wie auch die Selbstreflexion ausschlaggebend sind für die hohe Qualität der Beratungsleitungen bei ReMed. Über dieses Vorgehen wird einerseits den Bedürfnissen der Ratsuchenden Rechnung getragen, andererseits wird zentralen Aspekten der Qualitätssicherung von Beratungsangeboten Folge geleistet. Dieser Weg sollte auch künftig beibehalten werden [1].

\section{ReMed - Artikel und Informationen}

In der Schweizerischen Ärztezeitung wurden wiederum zwei Testimonials als Einblicke in die Beratungstätigkeit publiziert. Bedingt durch die Covid-19-Pandemie konnte ReMed nur an wenigen Anlässen präsent sein, viele Veranstaltungen wurden auf 2021 verschoben. Umso erfreulicher waren die Präsentationen für Medizinstudent(inn)en an der Universität Zürich und für Assistenz- und Oberärztinnen und -ärzte am Universitätsspital Zürich und im Spital Muri AG.

Besuchen Sie die Homepage www.swiss-remed.ch und finden Sie interessante Erfahrungsberichte und Dokumentationen sowie alle wertvollen Informationen über das Unterstützungsnetzwerk.

Das nationale Programm ReMed mit dem Leitungsausschuss und den Netzwerkmitgliedern wird auch im Jahr 2021 für Ärztinnen und Ärzte in Krisensituationen da sein. Die betroffenen Ärztinnen und Ärzte können über die 24-Stunden-Hotline (0800 0 73633) oder per remed[at]hin.ch Beratung und Hilfe anfordern.

\section{Literatur}

1 Hadorn L. Die Gesundheit der Ärztinnen und Ärzte im Fokus. Evaluation der ReMed-Unterstützungstätigkeit mit Fokus auf Beratungsqualität. Anonymisierte Fallauswertung. Arbeit zur Erlangung des Master of Public Health im Rahmen des Weiterbildungsstudiengangs Public Health der Universitäten Basel, Bern und Zürich; 2020 (bisher unveröffentlicht).

Bildnachweis

@ Dmytro Zinkevych | Dreamstime.com (Symbolbild) 